ARTICLE

https://doi.org/10.1038/s414.67-019-09062-8

OPEN

\title{
Intrinsic measurements of exciton transport in photovoltaic cells
}

\author{
Tao Zhang (1) ${ }^{1}$, Dana B. Dement ${ }^{1}$, Vivian E. Ferry ${ }^{1} \&$ Russell J. Holmes ${ }^{1}$
}

Organic photovoltaic cells are partiuclarly sensitive to exciton harvesting and are thus, a useful platform for the characterization of exciton diffusion. While device photocurrent spectroscopy can be used to extract the exciton diffusion length, this method is frequently limited by unknown interfacial recombination losses. We resolve this limitation and demonstrate a general, device-based photocurrent-ratio measurement to extract the intrinsic diffusion length. Since interfacial losses are not active layer specific, a ratio of the donor- and acceptor-material internal quantum efficiencies cancels this quantity. We further show that this measurement permits extraction of additional device-relevant information regarding exciton relaxation and charge separation processes. The generality of this method is demonstrated by measuring exciton transport for both luminescent and dark materials, as well as for small molecule and polymer active materials and semiconductor quantum dots. Thus, we demonstrate a broadly applicable device-based methodology to probe the intrinsic active material exciton diffusion length.

\footnotetext{
${ }^{1}$ Department of Chemical Engineering and Materials Science, University of Minnesota, Minneapolis, MN 55455, United States. Correspondence and requests for materials should be addressed to R.J.H. (email: rholmes@umn.edu)
} 
E xcitons are Coulombically-bound electron-hole pairs that serve as energetic intermediates in semiconductors. In many emerging semiconductor materials, the exciton and its properties feature prominently in determining device applications due to large binding energies that stabilize the exciton against thermally-driven dissociation ${ }^{1-8}$. Indeed, the high stability of the exciton has driven much research into the design of active materials and device architectures for organic light-emitting devices and organic photovoltaic cells (OPVs) ${ }^{9-15}$. Recently, there has also been growing interest in manipulating exciton transport at semiconductor heterointerfaces for devices like excitonic transistors ${ }^{16-18}$. For OPVs, in particular, excitons must migrate to a dissociating electron donor-acceptor (D-A) interface, making exciton transport a critical step towards efficient photoconver$\operatorname{sion}^{19-24}$. Consequently, substantial previous work has been directed at both the measurement and engineering of the exciton diffusion length $\left(L_{\mathrm{D}}\right)$. These efforts are essential as the scale of $L_{\mathrm{D}}$ dictates the optimal active layer thickness and domain size of $\mathrm{D}-\mathrm{A}$ blends in planar $(\mathrm{PHJ})$ and bulk heterojunction $(\mathrm{BHJ})$ OPVs, respectively ${ }^{25-29}$.

Probing exciton migration often relies on tracking the end-oflife products of excitons, including photons from exciton recombination and charge carriers from exciton dissociation ${ }^{30,31}$. For luminescent materials, $L_{\mathrm{D}}$ can be determined from a steady-state or time-resolved photoluminescence (PL)-based measurement $22,32,33$. Emitted photons reflect excitons that fail to reach the dissociating interface. While capable of yielding a materials-relevant value of $L_{\mathrm{D}}$, this method is only sensitive to diffusive states that can decay radiatively and therefore cannot be applied to materials forming weakly emissive or dark excitons ${ }^{34-36}$. Since many high-performing active materials are weakly luminescent or $\operatorname{dark}^{7,12,35,37,38}$, it is essential to develop more general means to accurately probe exciton transport in working devices.

A more general approach to extract $L_{\mathrm{D}}$ is by fitting the measured photocurrent (i.e., external quantum efficiency) spectrum of a bilayer $\mathrm{OPV}^{39-41}$. Device-based methods are attractive as they probe the $L_{\mathrm{D}}$ in a practical environment, as opposed to the often idealized structures utilized for PL measurements. This method is equally applicable to all excitons regardless of photoluminescence efficiency. Photoconversion can be considered in terms of four component processes, each with its own efficiency, namely light absorption $\left(\eta_{\mathrm{A}}\right)$, exciton diffusion and dissociation $\left(\eta_{\mathrm{D}}\right)$, charge transfer $(\mathrm{CT})$ state separation $\left(\eta_{\mathrm{CS}}\right)$, and free carrier collection $\left(\eta_{\mathrm{FC}}\right)$. As such, with knowledge of $\eta_{\mathrm{CS}}$ and $\eta_{\mathrm{FC}}$, coupled with an optical transfer matrix model for $\eta_{\mathrm{A}}, \eta_{\mathrm{D}}$ and $L_{\mathrm{D}}$ can be extracted by fitting with a diffusion equation. In practice, the values of $\eta_{\mathrm{CS}}$ and $\eta_{\mathrm{FC}}$ are not known, and frequently taken as unity or assumed to be fixed with changes in device architecture $39,42-45$. Thus, the extracted value of $L_{\mathrm{D}}$ is a lower bound to the actual, materials-specific $L_{\mathrm{D}}$. This limitation, coming from unknown geminate and non-geminate recombination losses, must be overcome in order to extract intrinsic, materials-specific values of $L_{\mathrm{D}}$.

Recently, we reported a technique based on transient photovoltage to overcome the non-geminate recombination limitation on extracting $L_{\mathrm{D}}$ from OPVs ${ }^{46,47}$. Photovoltage can be quantitatively translated into the number of free carriers stored within the device. For timescales much shorter than the carrier lifetime, the free carrier generation rate can be extracted from the initial photovoltage rise induced by illumination. The previously unknown $\eta_{\mathrm{FC}}$, therefore, can be quantitively measured by comparing the results of photovoltage and photocurrent measurements. Interestingly, $\eta_{\mathrm{FC}}$ is found to be near-unity at short-circuit for many PHJ systems. Consequently, an inability to quantify geminate recombination loss is the primary factor frustrating accurate extraction of $L_{\mathrm{D}}$ in devices.
Despite the presence of unknown recombination losses, it is possible to measure the intrinsic $L_{\mathrm{D}}$ from a device without the need to assume a value for $\eta_{\mathrm{Cs}}$. Previously, Vandewal et al. have shown that relaxed interfacial CT states (lowest energy) are the dominant source for free carrier generation in a wide range of $\mathrm{D}-\mathrm{A}$ systems ${ }^{48}$. This conclusion is further confirmed by recent work by Kurpiers et al. ${ }^{49}$. The value of $\eta_{\mathrm{CS}}$ is thus independent of whether a carrier originates from the donor or acceptor exciton. As such, a ratio of the donor and acceptor internal quantum efficiencies $\left(\eta_{\mathrm{IQE}}\right)$ cancels this unknown quantity and depends only on the exciton harvesting efficiencies $\left(\eta_{\mathrm{D}}\right)$ at short-circuit:

$$
\frac{\eta_{\mathrm{IQE}}^{\mathrm{D}}}{\eta_{\mathrm{IQE}}^{\mathrm{A}}}=\frac{\eta_{\mathrm{D}}^{\mathrm{D}} \eta_{\mathrm{CS}}}{\eta_{\mathrm{D}}^{\mathrm{A}} \eta_{\mathrm{CS}}}=\frac{\eta_{\mathrm{D}}^{\mathrm{D}}}{\eta_{\mathrm{D}}^{\mathrm{A}}}
$$

In order to model $\eta_{\mathrm{D}}$, exciton diffusion is treated with a onedimensional steady-state diffusion equation:

$$
0=D \frac{\partial^{2} n(x)}{\partial x^{2}}-\frac{n(x) D}{L_{\mathrm{D}}^{2}}+G(x)-n(x) k_{\mathrm{F}} \text { with } L_{\mathrm{D}}=\sqrt{D \tau}
$$

where $n$ is the exciton density, $D$ is the diffusion coefficient, $\tau$ is the exciton lifetime, and $G$ is the optical exciton generation rate calculated by transfer matrix modeling. For systems with a spectral overlap of donor emission and acceptor absorption, the rate of donor-acceptor Förster energy transfer (rate constant: $k_{\mathrm{F}}$ ) must be included. From the steady-state exciton density profile $n$ $(x)$, the $\eta_{\mathrm{D}}$ can be determined as:

$$
\eta_{\mathrm{D}}=1-\frac{\int n(x) / \tau \mathrm{d} x}{\int G(x) \mathrm{d} x}
$$

By fitting the $\eta_{\mathrm{D}}$ ratio in eq. (1) as a function of active layer thickness, $L_{\mathrm{D}}$ values for the donor and acceptor materials can be extracted simultaneously.

In this work, we apply the photocurrent-ratio method to extract $L_{\mathrm{D}}$ for both luminescent and dark organic semiconductors, including both small molecule and polymer active materials. We further demonstrate the broad utility of this approach by also extracting $L_{\mathrm{D}}$ for thin films of colloidal, inorganic semiconductor quantum dots. In order to compare the results of the photocurrent-ratio method to well-established photoluminescence quenching methods, we first consider the archetypical donor-acceptor pairing of boron subphthalocyanine chloride $(\mathrm{SubPc})$ and $\mathrm{C}_{60}$ (Fig. 1a), where both active materials are luminescent. This system shows negligible non-geminate recombination loss at short-circuit ${ }^{50,51}$, and prior work suggests that free carriers are likely generated from a single relaxed CT state $^{52}$, allowing eq. (1) to be applied. It is worth noting that in devices limited by non-geminate recombination, $\eta_{\mathrm{FC}}$ can be measured using transient photovoltage, leading to a modified, but equally useful form of eq. (1). For this luminescent pairing, we demonstrate that the values of $L_{\mathrm{D}}$ extracted using our devicebased method are in excellent agreement with those extracted using conventional PL-based measurements. Importantly, we further show that our device-based method yields the intrinsic material $L_{\mathrm{D}}$ even for devices that suffer from very low efficiency.

\section{Results}

Extracting $L_{\mathrm{D}}$ from a ratio of internal quantum efficiencies. To accurately measure $L_{\mathrm{D}}$ in bilayer OPVs, it is important to ensure that the only mechanism for exciton dissociation is at the donor-acceptor interface. Quenching at other interfaces or via bulk-ionization will lead to errors in the extracted $L_{\mathrm{D}}$. Figure $1 \mathrm{~b}$ shows the SubPc- $C_{60}$ PHJ OPV used for the measurement of $L_{D}$. The wide energy gap organic semiconductors 1,3-bis(carbazol-9yl)benzene (mCP) and bathocuproine (BCP) are employed as exciton blocking layers (EBLs) to prevent exciton quenching at 
a

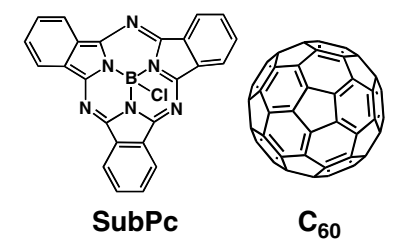

C
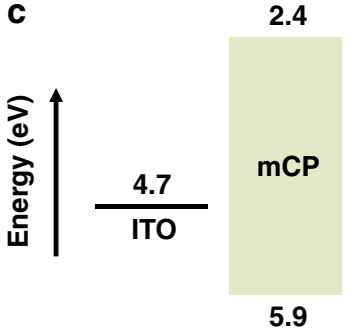

b

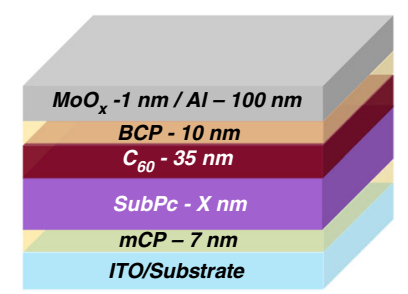

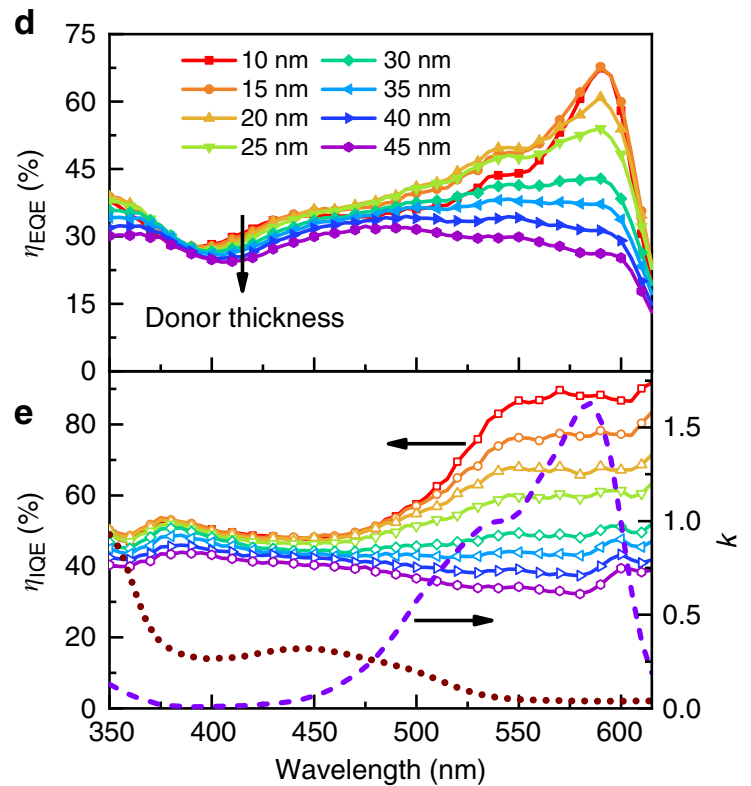

Fig. 1 Device architectures and quantum efficiency spectra for SubPc- $\mathrm{C}_{60}$ planar OPVs. a Molecular structure of SubPc and $\mathrm{C}_{60}$. $\mathbf{b}$ Device architecture for SubPc- $C_{60}$ planar OPVs. The SubPc thickness $X$ varies from $10 \mathrm{~nm}$ to $45 \mathrm{~nm}$. c Energy-level diagram for the devices in $\mathbf{b}$. $\mathbf{d}$ The $\eta_{\mathrm{EQE}} \mathrm{Spectra}$ measured at short-circuit as a function of SubPc layer thickness. e The $\eta_{\mathrm{IQE}}$ spectra calculated by dividing the $\eta_{\mathrm{EQE}}$ spectra in $\mathbf{d}$ by the $\eta_{\mathrm{A}}$ calculated using a transfer matrix model. The extinction coefficients $(k)$ of SubPc (purple dash line) and $\mathrm{C}_{60}$ (brown dot line) are also shown

active layer-electrode interfaces. The exclusion of quenching at the electrodes is critical for accurate measurements of $L_{\mathrm{D}}$ as exciton loss is observed when SubPc is in direct contact with ITO or the commonly used buffer layer $\mathrm{MoO}_{\mathrm{x}}$ (Supplementary Figure 1$)^{53}$. A 1-nm-thick layer of $\mathrm{MoO}_{\mathrm{x}}$ is used to increase the cathode work function and reduce the built-in electric field $\left(E_{\mathrm{bi}}\right)$ to exclude efficient exciton bulk-ionization processes at shortcircuit $^{54}$. This is especially important for the SubPc-C 60 system since both materials have been observed to show efficient free carrier generation in Schottky OPVs ${ }^{55,56}$. While the application of a forward bias can also be used to reduce $E_{\mathrm{bi}}$, the resulting carrier injection into the device could lead to exciton quenching, and an underestimate of $L_{\mathrm{D}}$. It is worth noting that while a reduced $E_{\mathrm{bi}}$ could hinder the separation of relaxed CT states at the donor-acceptor interface, we will demonstrate that this does not impact our measurement provided $\eta_{\mathrm{CS}}$ is identical for charges originating from both active materials.

Figure $1 \mathrm{~d}$ shows external quantum efficiency $\left(\eta_{\mathrm{EQE}}\right)$ spectra for SubPc- $\mathrm{C}_{60} \mathrm{PHJ}$ devices as a function of SubPc donor-layer thickness with the acceptor and buffer layer thicknesses held fixed. The $\eta_{\mathrm{A}}$ of the active materials calculated using a transfer matrix formalism (Supplementary Figure 2) allows the $\eta_{\mathrm{EQE}}$ spectra to be converted to $\eta_{\mathrm{IQE}}$ spectra (Fig. 1e $)^{41}$. To ensure the accuracy of the simulated $\eta_{\mathrm{A}}$, the result from transfer matrix modeling is checked against experimentally determined device reflectivity (through the anode, reflecting off the cathode) measured at an incident angle of $15^{\circ}$ to the substrate normal. The simulated 1-reflectivity $(R)$ spectra (absorption of the device stack) agree well with experimental results as a function of donor thickness (Supplementary Figure 3). For the device with a donor thickness of $10 \mathrm{~nm}$, the maximum $\eta_{\mathrm{EQE}}$ (at $\lambda=590 \mathrm{~nm}$, SubPc absorption) exceeds $65 \%$ and the corresponding $\eta_{\mathrm{IQE}}$ is about $85 \%$. This indicates that both exciton harvesting and charge collection are efficient in this device despite the reduced $E_{\mathrm{bi}}$. From the extinction coefficients $(\mathrm{k})$ of SubPc and $\mathrm{C}_{60}$, spectral regions of dominant absorption for both donor and acceptor can be isolated. Accordingly, for the construction of the $\eta_{\mathrm{IQE}}$ ratio, the individual $\eta_{\mathrm{IQE}}$ of the donor and acceptor are extracted from $\lambda=$ $575 \mathrm{~nm}$ (primarily SubPc absorption) and $\lambda=400 \mathrm{~nm}$ (primarily $\mathrm{C}_{60}$ absorption), respectively. Consistent with eq. (1), a $\eta_{\mathrm{D}}$ ratio $(\lambda=575 \mathrm{~nm}$ to $\lambda=400 \mathrm{~nm})$ is calculated as a function of SubPc thickness (Fig. 2a), which will be fit for $L_{D}$.

By fitting the $\eta_{\mathrm{D}}$ ratios in Fig. 2a, we simultaneously extract $L_{\mathrm{D}}$ values for SubPc and $C_{60}$ of $L_{\mathrm{D}}=(16.7 \pm 1.7) \mathrm{nm}$ and $L_{\mathrm{D}}=$ $(18.5 \pm 1.9) \mathrm{nm}$, respectively. Due to the spectral overlap of SubPc PL emission and $\mathrm{C}_{60}$ absorption, donor-acceptor Förster energy transfer is included when using eq. (2). We have previously measured the SubPc-C 60 Förster radius $\left(R_{0}\right)$ to be $2.1 \mathrm{~nm}$, consistent with the value of $2.1 \mathrm{~nm}$ calculated using Förster theory ${ }^{32}$. This value is short compared to the intrinsic $L_{\mathrm{D}}$ of SubPc, and hence does not significantly impact our measurement of $L_{\mathrm{D}}$ as diffusion is the dominant mechanism for harvesting in SubPc. As only the donor thickness is varied, the $L_{\mathrm{D}}$ of the donor and acceptor have different roles in determining the overall behavior of the $\eta_{\mathrm{D}}$ ratio as thickness changes (dash lines in Fig. 2a). For example, if the SubPc $L_{\mathrm{D}}$ is varied by $10 \%\left(\mathrm{C}_{60} L_{\mathrm{D}}\right.$ fixed), the resulting $\eta_{\mathrm{D}}$ ratios converge at small thickness while diverging at larger thickness, varying the shape of the dependence on thickness. Similar changes in the $\mathrm{C}_{60} L_{\mathrm{D}}\left(10 \%\right.$, SubPc $L_{\mathrm{D}}$ fixed) lead only to changes in the magnitude of the $\eta_{\mathrm{D}}$ ratio leaving the shape unchanged. To show this more explicitly, the values of $\eta_{\mathrm{D}}$ at $\lambda=575 \mathrm{~nm}$ and $400 \mathrm{~nm}$ are plotted as a function of donor thickness in Fig. $2 b$.

The observation of a constant acceptor $\eta_{\mathrm{D}}$ with increasing donor thickness offers some inherent advantages. First, the $L_{\mathrm{D}}$ of the donor and acceptor can be fit independently, as the shape of the $\eta_{\mathrm{D}}$ ratio (or normalized $\eta_{\mathrm{D}}$ ratio) only depends on donor $L_{\mathrm{D}}$. As such, any non-ideality in acceptor $L_{\mathrm{D}}$ measurement, such as exciton loss at the acceptor-cathode interface, would not reduce the accuracy of donor $L_{\mathrm{D}}$ provided free carriers are still only generated at the $\mathrm{D}-\mathrm{A}$ interface. In addition, the relative change in $\eta_{\mathrm{CS}}$ with donor thickness is directly reflected by the acceptor $\eta_{\mathrm{IQE}}$. Accordingly, the decrease in $\eta_{\mathrm{IQE}}(400 \mathrm{~nm})$ with $S$ SubPc thickness (Fig. 1e) indicates that a thicker donor layer leads to a lower $\eta_{\mathrm{CS}}$. 

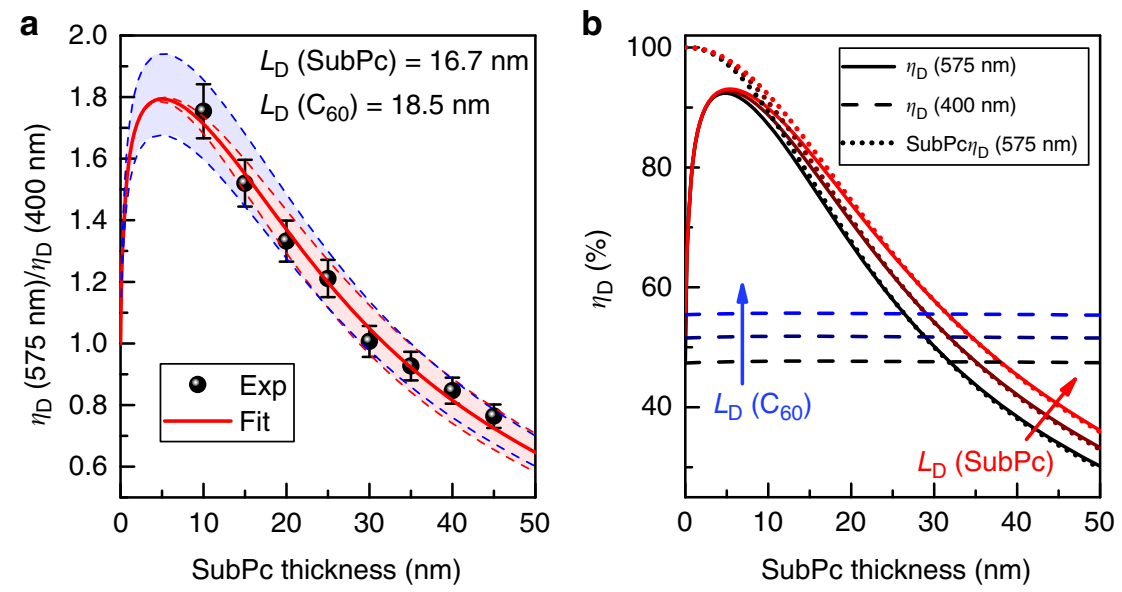

Fig. 2 Extracting $L_{D}$ from the thickness dependence of the diffusion efficiency ratio. a Diffusion efficiency ratio $(\lambda=575 \mathrm{~nm}$ to $\lambda=400 \mathrm{~nm})$ as a function of SubPc layer thickness with the corresponding exciton diffusion length $\left(L_{D}\right)$ extracted from the fit (solid red line) to the data. Red (blue) dash lines are simulated diffusion efficiency ratio curves for a $10 \%$ change in extracted SubPc or $C_{60} L_{D}$ while keeping the counterpart $L_{D}$ fixed. Error bars of diffusion efficiency ratio were determined based on the standard deviation of measured devices. $\mathbf{b}$ Solid lines are the diffusion efficiency at $\lambda=575 \mathrm{~nm}$ as a function of SubPc layer thickness for different SubPc $L_{D}(=15.0 / 16.7 / 18.3 \mathrm{~nm})$ and fixed $C_{60} L_{D}=18.5 \mathrm{~nm}$. Similarly, the horizontal dash lines are the diffusion efficiency at $\lambda=400 \mathrm{~nm}$ for different $C_{60} L_{D}(=16.7 / 18.5 / 20.3 \mathrm{~nm})$ and fixed SubPc $L_{D}=16.7 \mathrm{~nm}$. The diffusion efficiencies of SubPc at $\lambda=575 \mathrm{~nm}$ are also plotted as dot lines

This is likely due to an increase in bulk resistance of the donor layer that consumes more built-in voltage and thus reduces the electric field strength at D-A interface ${ }^{57}$.

Photoluminescence-based $\boldsymbol{L}_{\mathrm{D}}$ measurements. To determine whether the $L_{\mathrm{D}}$ extracted from the $\eta_{\mathrm{IQE}}$ ratio is intrinsic, conventional PL-based measurements are carried out for both SubPc and $\mathrm{C}_{60}$. In many previous studies, $\eta_{\mathrm{EQE}}$-based measurements of $L_{\mathrm{D}}$ have not been in agreement with those extracted from PL due to geminate recombination losses 46,50 , which are in general not negligible. If the recombination limitations are fully overcome in the method described here, these measurements should yield the same $L_{\mathrm{D}}$. For luminescent SubPc, the intrinsic $L_{\mathrm{D}}$ is extracted using thickness-dependent PL quenching and compared to the result obtained using the photocurrent-ratio method. In order to make a proper comparison, PL-quenching measurements are carried out on the identical device architecture used to measure $L_{\mathrm{D}}$ via $\eta_{\mathrm{IQE}}$ (Fig. 3a). Light having a wavelength of $\lambda=500 \mathrm{~nm}$ is incident through the ITO anode at an angle $\theta$ of $70^{\circ}$. As the emitted photons represent the excitons that fail to reach D-A interface, the PL intensity can be expressed as:

$$
\mathrm{PL}=\eta_{\mathrm{A}}\left(1-\eta_{\mathrm{D}}\right)\left(I_{\mathrm{in}} \eta_{\mathrm{PL}} \eta_{\text {det }}\right)
$$

where $I_{\mathrm{in}}$ is the incident photon flux, $\eta_{\mathrm{PL}}$ is the photoluminescence efficiency, $\eta_{\text {det }}$ is the detection efficiency for all the emitted photons. Typically, $L_{\mathrm{D}}$ is extracted by fitting a ratio of the integrated PL of a quenched sample (Q) and an unquenched sample (UQ). This PL ratio reflects the number of recombining excitons (i.e., not collected at the quenching interface) under steady-state:

$$
\frac{\mathrm{PL}^{\mathrm{Q}}}{\mathrm{PL}^{\mathrm{UQ}}}=\frac{\eta_{\mathrm{A}}^{\mathrm{Q}}\left(1-\eta_{\mathrm{D}}^{\mathrm{Q}}\right)\left(I_{\mathrm{in}} \eta_{\mathrm{PL}} \eta_{\mathrm{det}}\right)}{\eta_{\mathrm{A}}^{\mathrm{UQ}}\left(I_{\mathrm{in}} \eta_{\mathrm{PL}} \eta_{\mathrm{det}}\right)}=\frac{\eta_{\mathrm{A}}^{\mathrm{Q}}\left(1-\eta_{\mathrm{D}}^{\mathrm{Q}}\right)}{\eta_{\mathrm{A}}^{\mathrm{UQ}}}
$$

The $\eta_{\mathrm{A}}$ and $\eta_{\mathrm{D}}$ can be simulated using a transfer matrix formalism and a diffusion model as previously described. Here, the SubPc- $\mathrm{C}_{60} \mathrm{PHJ}$ devices in Fig. $1 \mathrm{~b}$ are used as quenched samples, with $\mathrm{C}_{60}$ acting as the quencher. A set of unquenched samples $\left(\eta_{\mathrm{D}}=0\right)$ are fabricated by replacing acceptor $\mathrm{C}_{60}$ with the exciton blocking material BCP. The resulting PL ratio is plotted in Fig. 3b and fit as a function of SubPc thickness, yielding a SubPc $L_{\mathrm{D}}$ of
$(16.6 \pm 2.0) \mathrm{nm}$. Clearly, the photocurrent-ratio and PL-based measurements conducted on the same set of devices show excellent agreement for the extracted $L_{\mathrm{D}}$. This agreement suggests that the charge recombination present in the device-based measurement does not limit our ability to extract $L_{\mathrm{D}}$.

To further verify the extracted value of $L_{\mathrm{D}}$, we use the $L_{\mathrm{D}}$ determined by the PL ratio to simulate the PL of quenched devices with eq. (4). Figure $3 c$ shows the integrated device PL of SubPc- $\mathrm{C}_{60} \mathrm{PHJ}$ devices as a function of SubPc thickness. Taking the prefactor $\left(I_{\mathrm{in}} \eta_{\mathrm{PL}} \eta_{\mathrm{det}}\right)$ as independent of SubPc thickness, the experimental results are in good agreement with the simulation using $L_{\mathrm{D}}=16.6 \mathrm{~nm}$ for SubPc.

In most reports of PL-based measurements of $L_{\mathrm{D}}$, actual device architectures are not utilized, replaced instead with simple film stacks. In this configuration, exciton dissociation due to bulkionization is negligible due to the absence of electrodes. If bulkionization in SubPc is negligible for the devices in Fig. 1b, $L_{\mathrm{D}}$ values for SubPc extracted from device and thin-film PL should agree. As shown in Fig. 3d, SubPc thin films are deposited on a 10-nm-thick $\mathrm{mCP} /$ glass substrate to ensure the SubPc film morphology is similar to the device-based measurement. The quenched films are capped with a 10-nm-thick layer of 1,4,5,8,9,11-hexaazatriphenylene hexacarbonitrile (HATCN) while the unquenched films are capped with a 10 -nm-thick layer of $\mathrm{mCP}^{58}$. Based on the thickness-dependent PL ratio of Fig. 3e, an intrinsic $L_{\mathrm{D}}$ of $(16.9 \pm 2.0) \mathrm{nm}$ is determined for SubPc. This value is larger than previous reports of the SubPc $L_{\mathrm{D}}$, which is around $10 \mathrm{~nm}^{32,59,60}$. However, previous studies typically deposit SubPc directly on an inorganic substrate such as glass as opposed to a wide energy gap organic buffer layer. By removing the 10$\mathrm{nm}$-thick mCP layer beneath the SubPc for unquenched samples, we observe a reduction in $\eta_{\mathrm{PL}}$, suggesting a difference in the morphology of SubPc on different substrates.

For weakly luminescent $\mathrm{C}_{60}$, a thin layer can result in a very low signal to noise ratio, which increases error for the extraction of $L_{\mathrm{D}}$. As such, we employ a 50 -nm-thick $\mathrm{C}_{60}$ layer and use spectrally resolved (SPR) PL quenching instead of thicknessdependent PL quenching ${ }^{59}$. We also use quartz as the substrate to further reduce the background noise. Figure $3 f$ shows $P L$ excitation spectra for the quenched and unquenched $\mathrm{C}_{60}$ films at an emission wavelength of $\lambda=750 \mathrm{~nm}$. The $\mathrm{PL}$ ratio is fit as a 
a

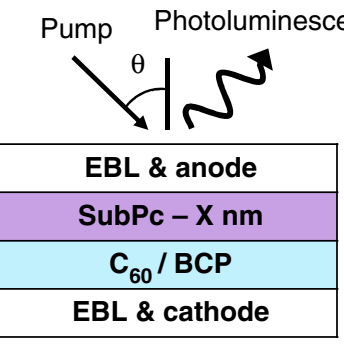

Planar devices b

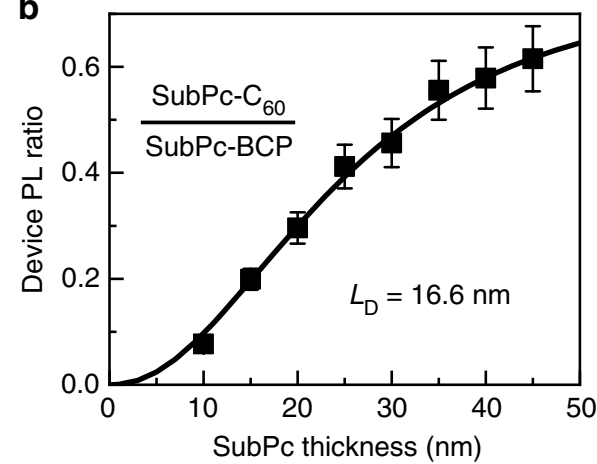

C

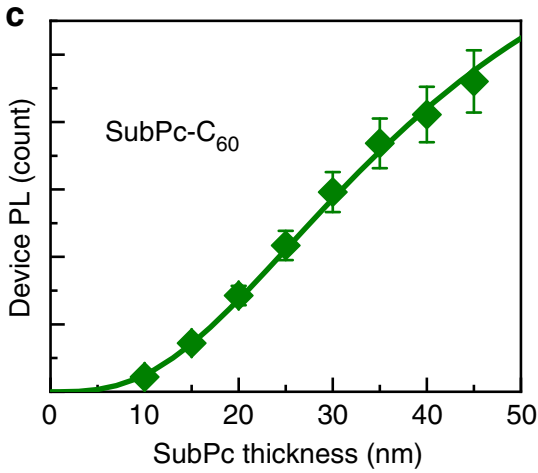

d

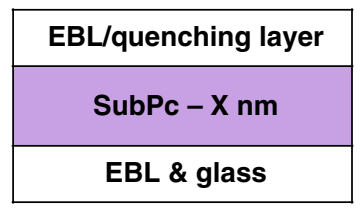

\begin{tabular}{|c|}
\hline EBL/quenching layer \\
\hline $\mathrm{C}_{60}-50 \mathrm{~nm}$ \\
\hline EBL \& Quartz \\
\hline SubPc/C ${ }_{60}$ thin films
\end{tabular}

e

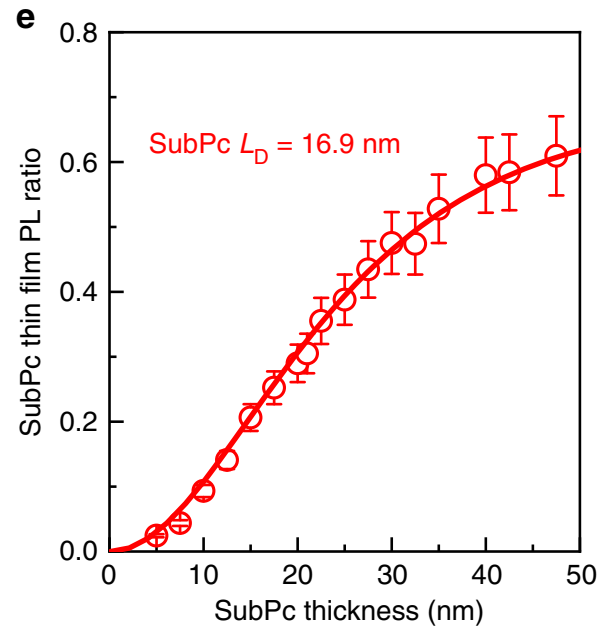

f

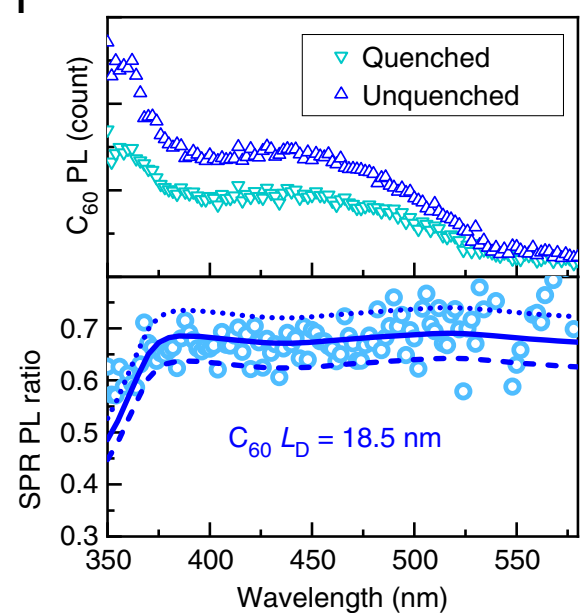

Fig. 3 Photoluminescence-based measurements of exciton diffusion length. a OPVs used for the extraction of $L_{D}$ via photoluminescence (PL) quenching having the structure: ITO/7 nm mCP/X (=10-45) nm SubPc/35 nm $\mathrm{C}_{60}$ (quenched) or $\mathrm{BCP}$ (unquenched) $/ 10 \mathrm{~nm} \mathrm{BCP/1} \mathrm{nm} \mathrm{MoO} / 100 \mathrm{~nm} \mathrm{Al.} \mathrm{The}$ quenched samples are the same set of devices used for internal quantum efficiency measurements. b PL ratio defined in eq. (5) versus SubPc thickness. c SubPc PL intensity of the quenched samples versus SubPc thickness. The solid line is fit of the data using eq. (4). Error bars reflect the run-to-run variation in PL intensity (standard deviation). d Architectures for conventional thin-film PL ratio $L_{D}$ measurements of SubPc and $\mathrm{C}_{60}$. SubPc architectures have the structure: glass $/ 10 \mathrm{~nm} \mathrm{mCP} / \mathrm{X}(=5.0-47.5) \mathrm{nm}$ SubPc/10 nm HATCN (quenched) or mCP (unquenched) while $\mathrm{C}_{60}$ architectures have the structure: quartz/10 nm BCP/50 nm C $60 / 10 \mathrm{~nm}$ HATCN (quenched) or BCP (unquenched). e The PL ratio of SubPc films as a function of SubPc thickness. f Excitation spectra (upper panel) for the quenched and unquenched $\mathrm{C}_{60}$ films at an emission wavelength of $750 \mathrm{~nm}$. The resulting spectrally resolved (SPR) PL ratio versus pump wavelength is shown in lower panel. SubPc films and devices are all pumped with $\lambda=500 \mathrm{~nm}$ light. The incident angle is $70^{\circ}$ for all incident light. Error bars for PL ratios are propagated from measured spectra and are proportional to the standard deviation in PL intensity measured multiple times

function of incident excitation wavelength, yielding an intrinsic $L_{\mathrm{D}}$ of $(18.5 \pm 3.0) \mathrm{nm}$ for $\mathrm{C}_{60}$. The excellent consistency of results between thin-film and device-based measurements further confirm that an intrinsic $L_{\mathrm{D}}$ can be accurately measured using the $\eta_{\mathrm{IQE}}$-based approach reported here. This agreement also suggests that there are no other diffusive dark states in SubPc and $\mathrm{C}_{60}$ that contribute to photocurrent since the photocurrent-ratio measurement probes both dark and emissive excitons while the PL-based measurement is only sensitive to emissive states.

Relaxation losses for high-energy excited states. With the $L_{\mathrm{D}}$ of SubPc and $\mathrm{C}_{60}$ extracted from device photocurrent spectra shown to be intrinsic, materials-relevant quantities, these values can be used to accurately simulate exciton diffusion and recombination losses in OPVs. The unknown geminate recombination loss $\left(\eta_{\mathrm{CS}}\right)$ that was circumvented in eq. (1) by taking a ratio of $\eta_{\mathrm{IQE}}$ values can now be calculated as the ratio of $\eta_{\mathrm{IQE}}$ to the calculated $\eta_{\mathrm{D}}$. Figure $4 \mathrm{a}$ shows the simulated $\eta_{\mathrm{EQE}}$ spectra for SubPc- $\mathrm{C}_{60} \mathrm{PHJ}$ devices using the $L_{\mathrm{D}}$ extracted in Fig. $1 \mathrm{~b}$, and the extracted $\eta_{\mathrm{CS}}$. The simulated $\eta_{\mathrm{EQE}}$ spectra agree well with experimental results except for the wavelength range of $\lambda=410-550 \mathrm{~nm}$. The observed
$\eta_{\mathrm{EQE}}$ overestimation indicates the presence of a photocurrent loss pathway prior to exciton diffusion, or stated differently, the exciton relaxation yield is not unity. It also worth noting the clear reduction in $\eta_{\mathrm{CS}}$ with increasing donor layer thickness, likely reflecting a reduction in $E_{\mathrm{bi}}$.

To identify whether the exciton relaxation loss is from SubPc or $\mathrm{C}_{60}$, a second donor-acceptor system of SubPc-NPD is examined where SubPc serves as the electron acceptor (or hole donor $)^{7}$. To ensure that the SubPc morphology is unchanged, an inverted device architecture is employed (ITO/7 $\mathrm{nm} \mathrm{mCP} / \mathrm{X} \mathrm{nm}$ SubPc/35 nm NPD/10 nm mCP/3 nm $\mathrm{MoO}_{\mathrm{x}} / 100 \mathrm{~nm} \mathrm{Al}$ ) so that SubPc is still deposited on $\mathrm{mCP}$. In these devices, the electron acceptor SubPc has low electron mobility, which can lead to significant recombination losses due to slow CT state separation $^{61}$. As shown in Supplementary Figure 4, the SubPc $\eta_{\mathrm{IQE}}$ (from $\lambda=525 \mathrm{~nm}$ ) and NPD $\eta_{\mathrm{IQE}}$ (from $\lambda=385 \mathrm{~nm}$ ) are both very low (less than $8 \%$ ). By fitting the $\eta_{\mathrm{D}}$ ratio as a function of SubPc thickness, an $L_{\mathrm{D}}$ of $(15.2 \pm 2.0) \mathrm{nm}$ is obtained for SubPc, close to the value extracted from the SubPc- $\mathrm{C}_{60}$ system despite significantly increased recombination losses. This result illustrates the power of the photocurrent-ratio technique, even in a lowefficiency device with substantial recombination loss, the correct, 


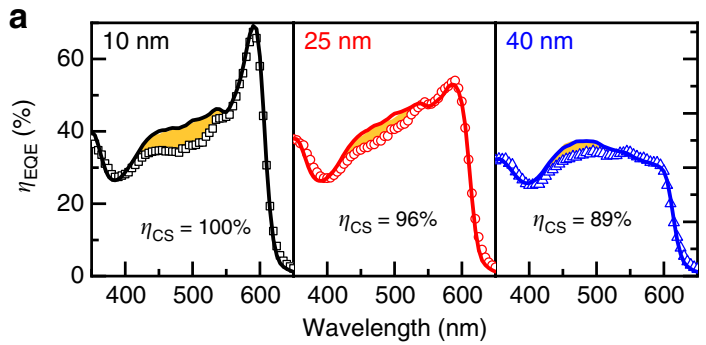

C
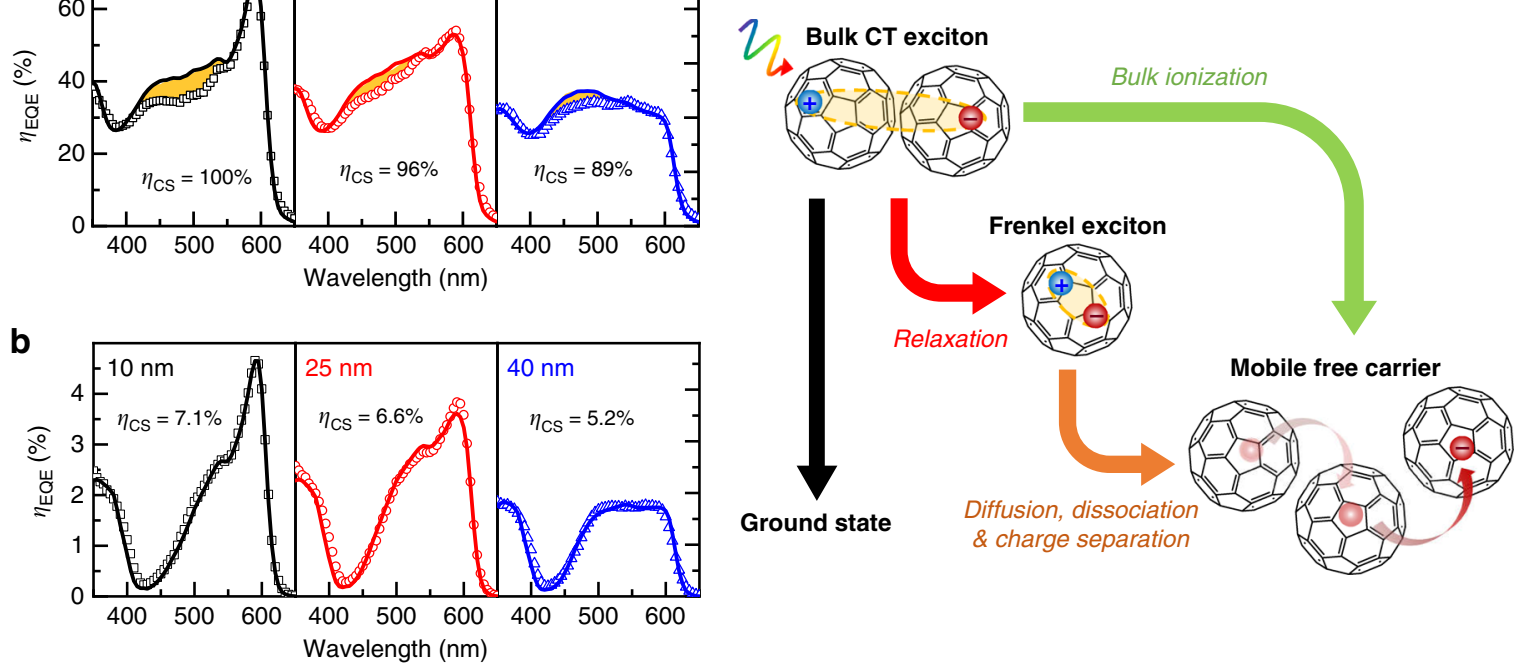

Fig. 4 Simulation of external quantum efficiency for extraction of recombination loss. a External quantum efficiency spectra for SubPc- $\mathrm{C}_{60}$ planar OPVs as a function of SubPc thickness (10, 25, and $40 \mathrm{~nm}$ ). Experimental results are shown in symbols while solid lines are simulated spectra. $\mathbf{b}$ The experimental (symbols) and simulated (solid lines) external quantum efficiency spectra of SubPc-NPD planar OPVs versus SubPc thickness. c Schematic of free carrier generation in $\mathrm{C}_{60}$ from a photoexcited bulk CT exciton. The bulk CT excitons absorb over the wavelength range of $\lambda=410$ to $550 \mathrm{~nm}$, while the low energy Frenkel excitons correspond to absorption at $\lambda$ greater than $550 \mathrm{~nm}$

intrinsic value of $L_{\mathrm{D}}$ is extracted. For the NPD dominant absorption region, an $\eta_{\mathrm{D}}(385 \mathrm{~nm})=47.1 \%$ is determined. Due to the large spectral overlap between NPD fluorescence and SubPc absorption, the dominant exciton harvesting mechanism within the NPD layer is Förster energy transfer to SubPc instead of exciton diffusion. In order to extract the $L_{\mathrm{D}}$ of NPD, a partner material should be chosen with reduced spectral overlap. Figure $4 \mathrm{~b}$ shows the simulated $\eta_{\mathrm{EQE}}$ spectra for SubPc-NPD $\mathrm{PHJ}$ devices based on the parameters extracted. Unlike the SubPc$\mathrm{C}_{60}$ case, simulated and experimental $\eta_{\mathrm{EQE}}$ spectra show broadband agreement as a function of SubPc thickness. This suggests that the exciton relaxation losses in SubPc- $\mathrm{C}_{60}$ devices originate only from excited states in $\mathrm{C}_{60}$.

Interestingly, the region $(\lambda=410-550 \mathrm{~nm})$ where exciton relaxation losses are observed is consistent with the broad absorption peak of $\mathrm{C}_{60}$ bulk CT excitons ${ }^{62}$. These bulk CT excitons can be photoexcited directly and their absorption $\left(\lambda_{\text {peak }}\right.$ around $450 \mathrm{~nm}$ ) has been observed to decrease drastically when diluting $\mathrm{C}_{60}$ in thin-film or solution ${ }^{13,63}$. This spectral agreement indicates that high energy bulk CT excitons do not uniformly relax to lowest energy Frenkel excitons in $\mathrm{C}_{60}$ despite their reasonably large energy difference (about $0.5 \mathrm{eV}$ ). For the $\mathrm{C}_{60}$ bulk CT excitons that fail to relax, they may still contribute to photocurrent through bulk-ionization (Fig. 4c), especially in devices with high built-in fields (see additional discussion in Supplementary Figure 5, Note 1). As such, both relaxation loss and bulk-ionization of bulk CT excitons must be considered in order to accurately simulate broadband $\eta_{\mathrm{EQE}}$ spectra of $\mathrm{C}_{60}$-based planar OPVs.

Elucidating exciton quenching and dissociation pathways. In order to accurately probe intrinsic exciton diffusion in OPVs, the architecture of Fig. $1 \mathrm{~b}$ was carefully designed to prevent exciton dissociation anywhere but at the D-A interface. As such, comparing its behavior to that of a conventional OPV allows additional exciton dissociation via electrode-organic interfaces and the built-in electric field to be quantified. For instance, removal of $\mathrm{mCP}$ between ITO and SubPc $(10 \mathrm{~nm})$ leads to a reduction in the SubPc exciton harvesting efficiency, permitting a quantification of quenching at the ITO interface. By inserting a wide gap interlayer to frustrate dissociation at the $\mathrm{D}-\mathrm{A}$ interface, the photocurrent contribution from bulk-ionization is also quantified. Combining these results with an exciton diffusion model and importantly, accurate measurements of $L_{\mathrm{D}}$, the fraction of photogenerated excitons consumed by various quenching, dissociation, and recombination pathways can be determined, as shown in Supplementary Figure 6. For $\lambda=590 \mathrm{~nm}$ (mainly SubPc), the fraction of excitons dissociated at the $\mathrm{D}-\mathrm{A}$ interface, quenched at the ITO-SubPc interface, and undergoing recombination within the donor layer are $73 \%, 21 \%$, and $6 \%$, respectively. For $\lambda=450 \mathrm{~nm}$ (mainly $\mathrm{C}_{60}$ ), $88 \%$ of $\mathrm{C}_{60}$ excitons relax to the lowest energy Frenkel states ( $47 \%$ dissociated at D-A interface and $41 \%$ recombining in the acceptor layer). Among the remaining $12 \%$ bulk CT excitons, $4 \%$ can contribute to photocurrent through bulk-ionization and $8 \%$ recombine prior to relaxation. These results suggest an important role for bulk-ionization via the builtin-field (especially for bulk CT excitons) in fullerene-based devices. This quantitative decoupling of photoconversion would not be accessible without the device-based $L_{\mathrm{D}}$ measurement reported here.

Dark small molecules and other excitonic materials. With the photocurrent-ratio method thoroughly vetted against conventional PL approaches for SubPc- $\mathrm{C}_{60}$, we investigate exciton transport in two dark small molecule materials, boron subnaphthalocyanine chloride (SubNc) and $\mathrm{C}_{70}$ (Fig. 5a). While these materials have been frequently utilized in high-efficiency OPVs, their intrinsic $L_{\mathrm{D}}$ has not been directly measured as they are inaccessible by PL-based techniques, $72,58,64$. Similar to the SubPc- $\mathrm{C}_{60}$ system, we employ a SubNc-C $\mathrm{C}_{70} \mathrm{PHJ}$ device with varying donor thickness to extract the intrinsic $L_{\mathrm{D}}$ of both dark materials (structure: ITO/8.5 $\mathrm{nm} \mathrm{mCP/X} \mathrm{(=9-40.5)} \mathrm{nm}$ SubNc/ $\left.27 \mathrm{~nm} \mathrm{C} 70 / 11 \mathrm{~nm} \mathrm{BCP} / 1 \mathrm{~nm} \mathrm{MoO}_{\mathrm{x}} / 100 \mathrm{~nm} \mathrm{Al}\right)$. Based on the extinction coefficients $(\mathrm{k})$ of SubNc and $\mathrm{C}_{70}$ shown in Fig. $5 \mathrm{~b}$, the individual $\eta_{\mathrm{IQE}}$ of the donor and acceptor can be extracted from $\lambda=685 \mathrm{~nm}$ (SubNc absorption peak) and $\lambda=430 \mathrm{~nm}$ (primarily $\mathrm{C}_{70}$ absorption), respectively. By fitting the donor-acceptor $\eta_{\mathrm{D}}$ ratio (determined as previously described) as a function of SubNc 
a<smiles></smiles><smiles>CC1(C)CCN2CCC(C)(C)c3cc(ccc3O2)C1=O</smiles>

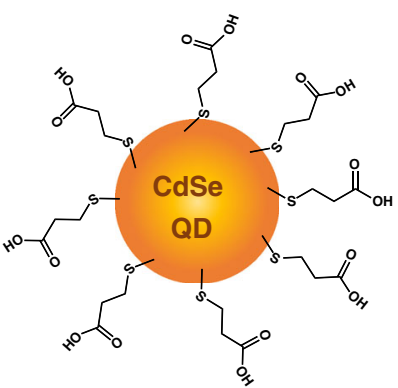

b

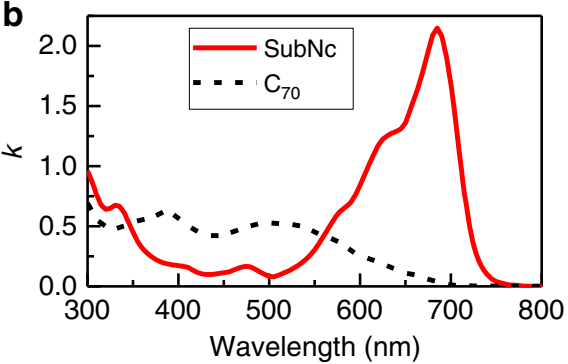

C

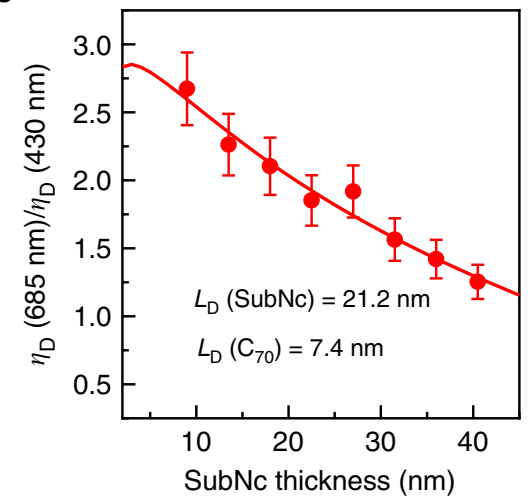

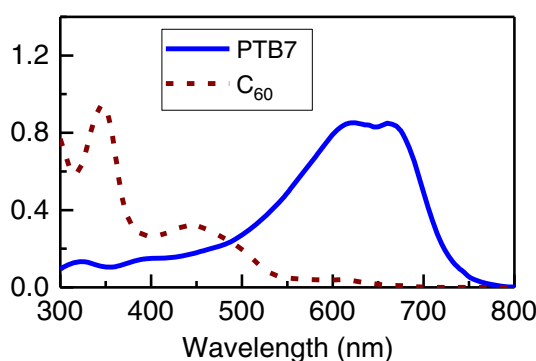

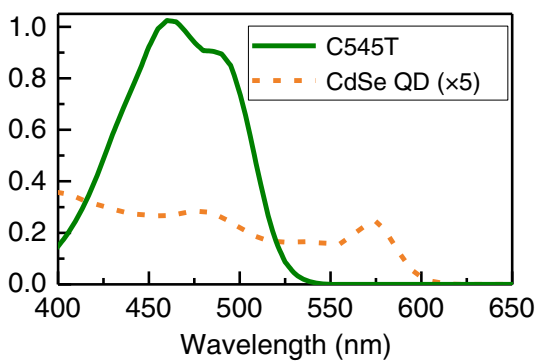

d

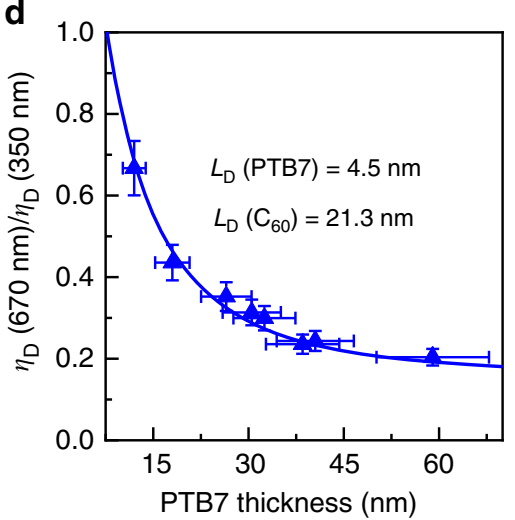

e

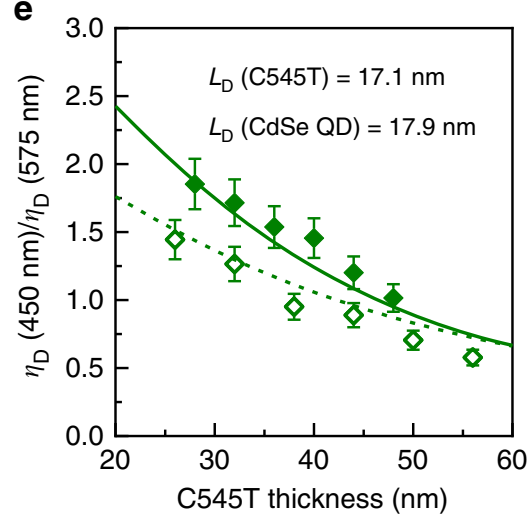

Fig. 5 Measuring $L_{D}$ of dark small molecules, polymers, and quantum dots. a Molecular structure of SubNc, PTB7, C545T and schematic of a CdSe quantum dot (QD) with 3-MPA ligands on the surface. $\mathbf{b}$ The extinction coefficients (k) of SubNc, $C_{70}$, PTB7, $C_{60}, C 545 T$, and CdSe QD thin films. The $k$ of QDs is shown with a 5-fold magnification. c Diffusion efficiency ratio $(\lambda=685 \mathrm{~nm}$ to $\lambda=430 \mathrm{~nm})$ as a function of SubNc layer thickness with the corresponding exciton diffusion length $\left(L_{D}\right)$ extracted from the fit (solid line) to the data. $\mathbf{d}$ Diffusion efficiency ratio $(\lambda=670 \mathrm{~nm}$ to $\lambda=350 \mathrm{~nm}$ ) as a function of PTB7 layer thickness. e Diffusion efficiency ratio $(\lambda=450 \mathrm{~nm}$ to $\lambda=575 \mathrm{~nm}$ ) as a function of C545T layer thickness and CdSe QD thickness (solid symbols: 66 $\mathrm{nm}$ QD layer; open symbols: $42 \mathrm{~nm}$ QD layer). The $L_{D}$ values are extracted by fitting both sets of data simultaneously (solid line: fit for $66 \mathrm{~nm}$ QD devices; dash line: fit for $42 \mathrm{~nm}$ QD devices). Error bars of diffusion efficiency ratio represent run-to-run variation (standard deviation) for the devices tested. Error bars of PTB7 thickness were standard deviation of multiple polymer thin films fabricated with the same procedures

thickness (Fig. 5c), we simultaneously extract an $L_{\mathrm{D}}$ of $(21.2 \pm 2.2)$ $\mathrm{nm}$ for SubNc and an $L_{\mathrm{D}}$ of $(7.4 \pm 0.8) \mathrm{nm}$ for $C_{70}$ (see Supplementary Figure 7 for $\eta_{\mathrm{EQE}}, \eta_{\mathrm{A}}$ and $\eta_{\mathrm{IQE}}$ spectra). The longer $L_{\mathrm{D}}$ of SubNc compared to SubPc $\left(L_{\mathrm{D}}=16.7 \mathrm{~nm}\right)$ is consistent with previous observations based on the PL of dilute solid solutions ${ }^{58}$. The extracted $L_{\mathrm{D}}$ for $\mathrm{C}_{70}$ is significantly smaller than that of $\mathrm{C}_{60}$ $\left(L_{\mathrm{D}}=18.5 \mathrm{~nm}\right)$. To better understand this difference, further work is needed to investigate differences in exciton lifetime and spin state between these fullerenes. Unlike the SubPc- $\mathrm{C}_{60}$ case, the simulated broadband $\eta_{\mathrm{EQE}}$ spectra of SubNc-C ${ }_{70}$ OPVs agree well with the experimental results, suggesting negligible exciton relaxation loss in $\mathrm{C}_{70}$. This result may reflect a reduced CT state character for excitons in $\mathrm{C}_{70}$.

To demonstrate the applicability of the photocurrent-ratio method in other excitonic systems, we also show two examples measuring exciton transport in solution processed PTB7 polymer films and inorganic CdSe quantum dot (QD) films. PTB7 is a high-performing polymer electron donor in OPVs, with demonstrated power efficiencies as high as $9.2 \%$ when combined with the fullerene acceptor $\mathrm{PC}_{71} \mathrm{BM}^{65}$. Here, we employ it as an electron-donor material paired with fullerene acceptor $\mathrm{C}_{60}$. Figure $5 \mathrm{~d}$ shows the PTB7- $\mathrm{C}_{60} \eta_{\mathrm{D}}$ ratio as a function of PTB7 thickness obtained from planar OPVs with a structure: ITO/2.5 $\mathrm{nm} \mathrm{HfO}_{2} / \mathrm{X}(=12-59) \mathrm{nm} \mathrm{PTB7} / 37 \mathrm{~nm} \mathrm{C} 60$ $\mathrm{MoO}_{\mathrm{x}} / 100 \mathrm{~nm} \mathrm{Al}$ (see Supplementary Figure 8 for $\eta_{\mathrm{EQE}}, \eta_{\mathrm{A}}$ and $\eta_{\mathrm{IQE}}$ spectra). A thin layer of wide energy gap $\mathrm{HfO}_{2}$ is utilized as an exciton blocking layer at ITO/donor interface (deposited via atomic layer deposition). The donor $\eta_{\mathrm{IQE}}$ is extracted from $\lambda=$ $670 \mathrm{~nm}$ (PTB7 absorption peak) while the acceptor $\eta_{\mathrm{IQE}}$ is extracted from $\lambda=350 \mathrm{~nm}\left(\mathrm{C}_{60}\right.$ Frenkel exciton absorption peak). As negligible exciton relaxation loss is observed at $\lambda=$ $350 \mathrm{~nm}$ in the previous SubPc- $\mathrm{C}_{60}$ case (Fig. 4a), it is assumed that $\mathrm{C}_{60}$ has $100 \%$ exciton relaxation yield at this wavelength. Fitting the $\eta_{\mathrm{D}}$ ratio in Fig. 5 d yields a short $L_{\mathrm{D}}$ of $(4.5 \pm 0.5) \mathrm{nm}$ 
for PTB7 and an $L_{\mathrm{D}}$ of $(21.3 \pm 2.2) \mathrm{nm}$ for $\mathrm{C}_{60}$. The $\mathrm{C}_{60} L_{\mathrm{D}}$ is similar to the value extracted from the SubPc- $\mathrm{C}_{60}$ system $(18.5 \mathrm{~nm})$.

For CdSe QD thin films, the QDs are zincblende nanocrystals (size $\sim 4 \mathrm{~nm}$ ) stabilized with 3-mercaptopropionic acid (3-MPA) after treatment via solid-state ligand exchange ${ }^{66}$. The resulting thin films have a very low extinction coefficient $(\mathrm{k}=0.05$ at excitonic peak, $\lambda=575 \mathrm{~nm}$ ), and are therefore paired with a wider energy gap small molecule 2,3,6,7-tetrahydro-1,1,7,7,-tetramethyl$1 \mathrm{H}, 5 \mathrm{H}, 11 \mathrm{H}-10$-(2-benzothiazolyl)quinolizino[9,9a,1gh]coumarin (C545T), which does not absorb at $\lambda=575 \mathrm{~nm}$. Wide gap materials $\mathrm{HfO}_{2}$ and di-[4-(N,N-di-p-tolyl-amino)-phenyl]cyclohexane (TAPC) are utilized as exciton blocking layers for CdSe QDs and C545T, respectively. The CdSe QD-C545T planar cell has an inverted device architecture: ITO/2.5 nm $\mathrm{HfO}_{2} / \mathrm{X}(=42$, 66) $\mathrm{nm}$ CdSe QD/Y (=26-56) nm C545T/11 nm TAPC/10 nm $\mathrm{MoO}_{\mathrm{x}} / 100 \mathrm{~nm} \mathrm{Al}$, where the CdSe QDs serves as the electron acceptor layer and photogenerated electrons are collected at ITO. It is again worth noting the ability of the photocurrent-ratio method in yielding intrinsic values of $L_{\mathrm{D}}$ even in devices with low efficiency. This allows active materials to be selected solely on the basis of realizing a minimal overlap in optical absorption. The QD film thickness is controlled by the number of cycles for the ligand exchange process ${ }^{66}$. Figure 5e shows the C545T-CdSe QD $\eta_{D}$ ratio as a function of C545T thickness for two different QD film thicknesses (see Supplementary Figure 9 for broadband $\eta_{\mathrm{EQE}}, \eta_{\mathrm{A}}$ and $\eta_{\mathrm{IQE}}$ spectra). The donor $\eta_{\mathrm{IQE}}$ is extracted at $\lambda=450 \mathrm{~nm}$ (C545T absorption peak) while the acceptor $\eta_{\mathrm{IQE}}$ is extracted at $\lambda=575 \mathrm{~nm}$ (QD excitonic absorption peak). Results for devices with QD layer thickness of $42 \mathrm{~nm}$ and $66 \mathrm{~nm}$ are fitted simultaneously, yielding an $L_{\mathrm{D}}$ of $(17.9 \pm 3.6) \mathrm{nm}$ for the CdSe QDs and an $L_{\mathrm{D}}$ of $(17.1 \pm 3.4) \mathrm{nm}$ for C545T. It is worth noting that the $L_{\mathrm{D}}$ of C545T extracted here is consistent with separate PLbased measurements we have carried out $(16.7 \mathrm{~nm})$. If instead the $\eta_{\mathrm{D}}$ ratio is fit for each QD thickness, the $42 \mathrm{~nm}(66 \mathrm{~nm})$ QDC545T devices yield an $L_{\mathrm{D}}$ of $18.7 \mathrm{~nm}(15.5 \mathrm{~nm})$ and $19.8 \mathrm{~nm}$ $(16.8 \mathrm{~nm})$ for the QDs and C545T, respectively. The $L_{\mathrm{D}}$ of CdSe QDs extracted here is smaller than that previously reported for CdSe core-shell counterparts (20 to $40 \mathrm{~nm})^{67,68}$, likely reflecting that a wide gap shell is critical to frustrate exciton decay and enhance exciton transport for CdSe QDs.

\section{Discussion}

We demonstrate a simple photocurrent-ratio method to measure the intrinsic $L_{\mathrm{D}}$ of excitonic semiconductors in bilayer photovoltaic cells. The impact of often unknown recombination losses is negated when taking a ratio of donor and acceptor $\eta_{\mathrm{IQE}}$. For the luminescent organic pairing $\mathrm{SubPc}$ and $\mathrm{C}_{60}$, their $L_{\mathrm{D}}$ extracted using this method are in excellent agreement with those extracted from conventional PL-quenching measurements, a direct result of the ability to remove the role of unknown geminate recombination losses. With knowledge of the intrinsic values of $L_{\mathrm{D}}$, we are able to investigate the potential for relaxation losses, which are typically overlooked in device-based studies of $L_{\mathrm{D}}$. Indeed, bulk CT excitons generated in $\mathrm{C}_{60}$ have non-unity relaxation yield to Frenkel states and can generate free carriers directly through bulk-ionization. The generality of this photocurrent-ratio method is further demonstrated by extracting $L_{\mathrm{D}}$ for the dark small molecule SubNc, $\mathrm{C}_{70}$, the highperformance polymer PTB7, as well as for CdSe quantum dot thin films. Thus, we demonstrate a straightforward measurement for the extraction of $L_{\mathrm{D}}$ that is equally applicable to both luminescent and dark excitonic materials capable of being integrated into a bilayer photovoltaic cell.

\section{Methods}

Chemicals. For this study, SubPc (99\%), mCP (99\%), TPBi (99\%), C545T (99\%), TAPC (99\%), and PTB7 (Mw > 50,000) were obtained from Lumtec Inc., $\mathrm{C}_{60}(99 \%)$ and $\mathrm{C}_{70}(99 \%)$ were obtained from MER Corporation, $\mathrm{MoO}_{3}(99 \%)$ and $\mathrm{BCP}$ (98\%) were obtained from Alfa Aesar, chloroform (99\%), 3-mercaptopropionic acid (99\%), octane (98\%), oleic acid (tech. grade 90\%) acetonitrile (99.5\%), and methyl acetate (anhydrous) were obtained from Sigma-Aldrich, selenium dioxide $(99.8 \%)$ and 1-octadecene (90\%) were obtained from Acros Organics. All materials above were used as received. The NPD (99\%) was synthesized by the Dow Chemical Company and purified once by temperature-gradient sublimation ${ }^{69}$. Cadmium myristate was prepared on a multi-gram scale according to the method reported by Chen et al. ${ }^{70}$.

Synthesis of zincblende CdSe QDs. Zincblende CdSe QDs with oleic acid capping ligands were synthesized as previously reported ${ }^{66}$, based off the original synthesis by Chen and et al. ${ }^{70}$. Briefly, 1 -octadecene $(63 \mathrm{~mL})$, cadmium myristate $(570 \mathrm{mg})$ and selenium dioxide $(110 \mathrm{mg})$ were added to a 3-neck round bottom flask and degassed at $75^{\circ} \mathrm{C}$ for $1 \mathrm{~h}$. After degassing, the reaction mixture was heated to $240^{\circ} \mathrm{C}$, at which point $1 \mathrm{~mL}$ of oleic acid was added dropwise to the solution once a red color was achieved. The reaction was allowed to proceed for $15 \mathrm{~min}$ and then cooled to room temperature for purification by centrifugation in methyl acetate. The resulting precipitate was dispersed in octane and filtered using a 0.2 um PTFE syringe filter prior to film fabrication. Resulting solution concentration was $\sim 25 \mathrm{~g} \mathrm{~L}^{-1}$

Thin-film and device preparation. Organic photovoltaic cells were fabricated using indium-tin-oxide (ITO)-coated glass substrates with a sheet resistance of 8-12 $\Omega$ per $\square$. Substrates were cleaned in tergitol solution and in organic solvents and treated in UV-Ozone ambient for $10 \mathrm{~min}$ prior to thin-film deposition. All polymer and QD films were spin-coated onto an $\mathrm{HfO}_{2}$-coated ITO substrate. The $\mathrm{HfO}_{2}$ was deposited via atomic layer deposition at $200^{\circ} \mathrm{C}$. The polymer PTB7 was first dispersed in chloroform and then spin-coated at $3000 \mathrm{rpm}$ for $45 \mathrm{~s}$. The obtained samples were annealed in $\mathrm{N}_{2}$ at $140{ }^{\circ} \mathrm{C}$ for $10 \mathrm{~min}$. The polymer film thickness was controlled via the concentration of the PTB7 solution $\left(2-10 \mathrm{~g} \mathrm{~L}^{-1}\right)$. Spin-coating and buildup of QD film thickness were realized using a solid-state ligand exchange procedure. CdSe QDs dispersed in octane were spin-coated at $1200 \mathrm{rpm}$ for $30 \mathrm{~s}$ and then $6000 \mathrm{rpm}$ for $60 \mathrm{~s}$. The latter process is found to improve film uniformity and reproducibility. Next, 3-MPA ( $1 \% \mathrm{v} / \mathrm{v})$ in acetonitrile was first deposited onto the film and allowed to sit for $10 \mathrm{~s}$ to facilitate the ligand exchange. The sample was then spun again at $1200 \mathrm{rpm}$ for $30 \mathrm{~s}$. Finally, the film was rinsed with pure acetonitrile by spinning at $6000 \mathrm{rpm}$ for $60 \mathrm{~s}$. This process was repeated 3-4 times until the desired QD film thickness was achieved. All other layers were deposited at room temperature by high vacuum thermal evaporation at a pressure of less than $8 \times 10^{-7}$ Torr. The active area of the obtained device is 0.785 $\mathrm{mm}^{2}$, defined by the cathode area.

Optoelectronic characterization. Photoluminescence quenching data were recorded using a Photon Technology International QuantaMaster Fluorometer. All photoluminescence measurements were performed under $\mathrm{N}_{2}$ purge. Photoluminescence quenching measurements were made at an incident angle of $70^{\circ}$ to the substrate normal. External quantum efficiency measurements were collected by measuring the photocurrent under monochromatic light illumination using a $300 \mathrm{~W}$ Oriel Xe lamp, a monochromator, an optical chopper wheel, and an SR810 lock-in amplifier. Error bars for $L_{\mathrm{D}}$ represent a $95 \%$ confidence interval extracted from fitting parameters. All film thicknesses, reflectivity spectra were measured with a J. A. Woollam spectroscopic ellipsometer. The device reflectivity measurements were made at an incident angle of $15^{\circ}$ to the substrate normal. Film thicknesses were fit using a Cauchy model. For simulations of device absorption efficiency optical constants of organic and QD thin films were extracted by fitting transmission (at normal incidence) and reflection (incident angle: $15^{\circ}$ ) simultaneously with a transfer matrix formalism (provided in Supplementary Figure 10).

\section{Data availability}

The data presented within this manuscript are available from the corresponding author upon reasonable request.

Received: 29 June 2018 Accepted: 13 February 2019

Published online: 11 March 2019

\section{References}

1. Ye, Z. et al. Probing excitonic dark states in single-layer tungsten disulphide. Nature 513, 214 (2014).

2. Ye, Y. et al. Monolayer excitonic laser. Nat. Photonics 9, 733 (2015). 
3. Blancon, J.-C. et al. Extremely efficient internal exciton dissociation through edge states in layered 2d perovskites. Science 355, 1288-1292 (2017).

4. Zhang, Q., Chu, L., Zhou, F., Ji, W. \& Eda, G. Excitonic properties of chemically synthesized $2 \mathrm{D}$ organic-inorganic hybrid perovskite nanosheets. Adv. Mater. 30, 1704055 (2018).

5. Milichko, V. A. et al. Van der waals metal-organic framework as an excitonic material for advanced photonics. Adv. Mater. 29, 1606034 (2017).

6. Najafov, H., Lee, B., Zhou, Q., Feldman, L. C. \& Podzorov, V. Observation of long-range exciton diffusion in highly ordered organic semiconductors. Nat. Mater. 9, 938 (2010).

7. Cnops, K. et al. $8.4 \%$ efficient fullerene-free organic solar cells exploiting longrange exciton energy transfer. Nat. Commun. 5, 3406 (2014).

8. Shirasaki, Y., Supran, G. J., Bawendi, M. G. \& Bulović, V. Emergence of colloidal quantum-dot light-emitting technologies. Nat. Photonics 7, 13 (2013).

9. Sun, Y. et al. Management of singlet and triplet excitons for efficient white organic light-emitting devices. Nature 440, 908-912 (2006).

10. Coburn, C., Lee, J. \& Forrest, S. R. Charge balance and exciton confinement in phosphorescent organic light emitting diodes. Adv. Opt. Mater. 4, 889-895 (2016).

11. Zhang, S., Qin, Y., Zhu, J. \& Hou, J. Over $14 \%$ efficiency in polymer solar cells enabled by a chlorinated polymer donor. Adv. Mater. 30, 1800868 (2018).

12. Che, X., Li, Y., Qu, Y. \& Forrest, S. R. High fabrication yield organic tandem photovoltaics combining vacuum- and solution-processed subcells with $15 \%$ efficiency. Nat. Energy 3, 422-427 (2018)

13. Che, X., Xiao, X., Zimmerman, J. D., Fan, D. \& Forrest, S. R. High-efficiency, vacuum-deposited, small-molecule organic tandem and triple-junction photovoltaic cells. Adv. Energy Mater. 4, 1400568 (2014).

14. Zhao, W. et al. Molecular optimization enables over $13 \%$ efficiency in organic solar cells. J. Am. Chem. Soc. 139, 7148-7151 (2017).

15. Lin, Y. et al. Mapping polymer donors toward high-efficiency fullerene free organic solar cells. Adv. Mater. 29, 1604155 (2017).

16. Deotare, P. B. et al. Nanoscale transport of charge-transfer states in organic donor-acceptor blends. Nat. Mater. 14, 1130-1134 (2015).

17. High, A. A., Novitskaya, E. E., Butov, L. V., Hanson, M. \& Gossard, A. C. Control of exciton fluxes in an excitonic integrated circuit. Science 321, 229-231 (2008).

18. Unuchek, D. et al. Room-temperature electrical control of exciton flux in a van der waals heterostructure. Nature 560, 340-344 (2018).

19. Lin, J. D. et al. Systematic study of exciton diffusion length in organic semiconductors by six experimental methods. Mater. Horiz. 1, 280-285 (2014).

20. Mikhnenko, O. V., Blom, P. W. \& Nguyen, T.-Q. Exciton diffusion in organic semiconductors. Energy Environ. Sci. 8, 1867-1888 (2015).

21. Menke, S. M. \& Holmes, R. J. Exciton diffusion in organic photovoltaic cells. Energy Environ. Sci. 7, 499-512 (2014).

22. Hedley, G. J., Ruseckas, A. \& Samuel, I. D. Light harvesting for organic photovoltaics. Chem. Rev. 117, 796-837 (2016).

23. Scharber, M. C. et al. Design rules for donors in bulk-heterojunction solar cells -towards 10\% energy-conversion efficiency. Adv. Mater. 18, 789-794 (2006).

24. Kippelen, B. \& Brédas, J.-L. Organic photovoltaics. Energy Environ. Sci. 2, 251-261 (2009).

25. Park, S. H. et al. Bulk heterojunction solar cells with internal quantum efficiency approaching 100\%. Nat. Photonics 3, 297 (2009).

26. Mayer, A. C., Scully, S. R., Hardin, B. E., Rowell, M. W. \& McGehee, M. D. Polymer-based solar cells. Mater. Today 10, 28-33 (2007).

27. Halls, J. J., Pichler, K., Friend, R. H., Moratti, S. \& Holmes, A. Exciton diffusion and dissociation in a poly (p-phenylenevinylene)/ $\mathrm{C}_{60}$ heterojunction photovoltaic cell. Appl. Phys. Lett. 68, 3120-3122 (1996).

28. Tamai, Y., Ohkita, H., Benten, H. \& Ito, S. Exciton diffusion in conjugated polymers: from fundamental understanding to improvement in photovoltaic conversion efficiency. J. Phys. Chem. Lett. 6, 3417-3428 (2015).

29. Westenhoff, S., Howard, I. A. \& Friend, R. H. Probing the morphology and energy landscape of blends of conjugated polymers with sub-10 $\mathrm{nm}$ resolution. Phys. Rev. Lett. 101, 016102 (2008).

30. Penwell, S. B., Ginsberg, L. D. S., Noriega, R. \& Ginsberg, N. S. Resolving ultrafast exciton migration in organic solids at the nanoscale. Nat. Mater. 16, 1136-1141 (2017).

31. Kose, M. E. et al. Exciton migration in conjugated dendrimers: ajoint experimental and theoretical study. Chemphyschem 10, 3285-3294 (2009).

32. Luhman, W. A. \& Holmes, R. J. Investigation of energy transfer in organic photovoltaic cells and impact on exciton diffusion length measurements. Adv. Funct. Mater. 21, 764-771 (2011).

33. Mikhnenko, O. V. et al. Exciton diffusion length in narrow bandgap polymers. Energy Environ. Sci. 5, 6960-6965 (2012).

34. Thompson, N. J. et al. Energy harvesting of non-emissive triplet excitons in tetracene by emissive PbS nanocrystals. Nat. Mater. 13, 1039 (2014).
35. Congreve, D. N. et al. External quantum efficiency above $100 \%$ in a singletexciton-fission-based organic photovoltaic cell. Science 340, 334-337 (2013).

36. Efros, A. L. et al. Band-edge exciton in quantum dots of semiconductors with a degenerate valence band: dark and bright exciton states. Phys. Rev. B 54, 4843 (1996).

37. Zou, Y., Holst, J., Zhang, Y. \& Holmes, R. J. 7.9\% efficient vapor-deposited organic photovoltaic cells based on a simple bulk heterojunction. J. Mater. Chem. A 2, 12397 (2014).

38. Bergemann K. J., Liu X., Panda A. \& Forrest S. R. Singlets lead to photogeneration in $\mathrm{C}_{60}$-based organic heterojunctions. Phys. Rev. B 92, 035408 (2015)

39. Peumans, P., Yakimov, A. \& Forrest, S. R. Small molecular weight organic thin-film photodetectors and solar cells. J. Appl. Phys. 93, 3693 (2003).

40. Luhman, W. A. \& Holmes, R. J. Enhanced exciton diffusion in an organic photovoltaic cell by energy transfer using a phosphorescent sensitizer. Appl. Phys. Lett. 94, 153304 (2009).

41. Pettersson, L. A. A., Roman, L. S. \& Inganas, O. Modeling photocurrent action spectra of photovoltaic devices based on organic thin films. J. Appl. Phys. 86, 487-496 (1999).

42. Terao, Y., Sasabe, H. \& Adachi, C. Correlation of hole mobility, exciton diffusion length, and solar cell characteristics in phthalocyanine/fullerene organic solar cells. Appl. Phys. Lett. 90, 103515 (2007)

43. Kim, I. et al. Efficient organic solar cells based on planar metallophthalocyanines. Chem. Mater. 21, 4256-4260 (2009).

44. Guide, M. et al. Effect of copper metalation of tetrabenzoporphyrin donor material on organic solar cell performance. J. Mater. Chem. A 2, 7890-7896 (2014).

45. Siegmund, B. et al. Exciton diffusion length and charge extraction yield in organic bilayer solar cells. Adv. Mater. 29, 1604424 (2017).

46. Mullenbach, T. K., Curtin, I. J., Zhang, T. \& Holmes, R. J. Probing dark exciton diffusion using photovoltage. Nat. Commun. 8, 14215 (2017).

47. Zhang, T. \& Holmes, R. J. Photovoltage as a quantitative probe of carrier generation and recombination in organic photovoltaic cells. J. Mater. Chem. C 5, 11885-11891 (2017).

48. Vandewal, K. et al. Efficient charge generation by relaxed charge-transfer states at organic interfaces. Nat. Mater. 13, 63 (2014).

49. Kurpiers, J. et al. Probing the pathways of free charge generation in organic bulk heterojunction solar cells. Nat. Commun. 9, 2038 (2018).

50. Curtin I. J. \& Holmes R. J. Decoupling photocurrent loss mechanisms in photovoltaic cells using complementary measurements of exciton diffusion. Adv. Energy Mater. 8, 1702339 (2018). https://doi.org/10.1002/aenm.201702339.

51. Credgington, D., Liu, S.-W., Nelson, J. \& Durrant, J. R. In situ measurement of energy level shifts and recombination rates in subphthalocyanine/ $\mathrm{C}_{60}$ bilayer solar cells. J. Phys. Chem. C 118, 22858-22864 (2014).

52. Pandey, R. \& Holmes, R. J. Characterizing the charge collection efficiency in bulk heterojunction organic photovoltaic cells. Appl. Phys. Lett. 100, 083303 (2012).

53. Zou, Y. \& Holmes, R. J. Influence of a $\mathrm{MoO}_{\mathrm{x}}$ interlayer on the open-circuit voltage in organic photovoltaic cells. Appl. Phys. Lett. 103, 053302 (2013).

54. Yi, Y. et al. The interface state assisted charge transport at the $\mathrm{MoO}(3) / \mathrm{metal}$ interface. J. Chem. Phys. 130, 094704 (2009).

55. Zou, Y. \& Holmes, R. J. The role of exciton ionization processes in bulk heterojunction organic photovoltaic cells. Adv. Energy Mater. 5, 1500019 (2015).

56. Chandran, H. T. et al. Direct free carrier photogeneration in single layer and stacked organic photovoltaic devices. Adv. Mater. 29, 1606909 (2017).

57. Proctor, C. M., Kuik, M. \& Nguyen, T.-Q. Charge carrier recombination in organic solar cells. Prog. Polym. Sci. 38, 1941-1960 (2013).

58. Menke, S. M. \& Holmes, R. J. Energy-cascade organic photovoltaic devices incorporating a host-guest architecture. ACS Appl. Mater. Interfaces 7, 2912-2918 (2015).

59. Lunt, R. R., Giebink, N. C., Belak, A. A., Benziger, J. B. \& Forrest, S. R. Exciton diffusion lengths of organic semiconductor thin films measured by spectrally resolved photoluminescence quenching. J. Appl. Phys. 105, 053711 (2009).

60. Menke, S. M., Luhman, W. A. \& Holmes, R. J. Tailored exciton diffusion in organic photovoltaic cells for enhanced power conversion efficiency. Nat. Mater. 12, 152-157 (2013).

61. Pandey, R., Gunawan, A. A., Mkhoyan, K. A. \& Holmes, R. J. Efficient organic photovoltaic cells based on nanocrystalline mixtures of boron subphthalocyanine chloride and $\mathrm{C}_{60}$. Adv. Funct. Mater. 22, 617-624 (2012)

62. Hahn, T. et al. Role of intrinsic photogeneration in single layer and bilayer solar cells with c60 and pcbm. J. Phys. Chem. C 120, 25083-25091 (2016).

63. Ishibashi, Y., Arinishi, M., Katayama, T., Miyasaka, H. \& Asahi, T. Femtosecond excited-state dynamics of fullerene- $\mathrm{C}_{60}$ nanoparticles in water. Phys. Chem. Chem. Phys. 20, 958-966 (2018).

64. Li, Y. et al. Near-infrared ternary tandem solar cells. Adv. Mater. 30, 1804416 (2018). 
65. He, Z. et al. Enhanced power-conversion efficiency in polymer solar cells using an inverted device structure. Nat. Photonics 6, 591 (2012).

66. Dement, D. B., Puri, M. \& Ferry, V. E. Determining the complex refractive index of neat CdSe/CdS quantum dot films. J. Phys. Chem. C 122, 21557-21568 (2018).

67. Akselrod, G. M. et al. Subdiffusive exciton transport in quantum dot solids. Nano. Lett. 14, 3556-3562 (2014).

68. Lee, E. M. Y. \& Tisdale, W. A. Determination of exciton diffusion length by transient photoluminescence quenching and its application to quantum dot films. J. Phys. Chem. C 119, 9005-9015 (2015).

69. Curtin, I. J., Blaylock, D. W. \& Holmes, R. J. Role of impurities in determining the exciton diffusion length in organic semiconductors. Appl. Phys. Lett. 108, 163301 (2016).

70. Chen, O. et al. Synthesis of metal-selenide nanocrystals using selenium dioxide as the selenium precursor. Angew. Chem. Int. Ed. 120, 8766-8769 (2008).

\section{Acknowledgements}

This work was supported by National Science Foundation (NSF) Electronics, Photonics, and Magnetic Devices under ECCS-1509121. T.Z. acknowledges support from a University of Minnesota Doctoral Dissertation Fellowship. The authors thank Dr. D. Wayne Blaylock at The Dow Chemical Company for synthesizing NPD, Deepesh Rai for measuring the C545T $L_{\mathrm{D}}$ via photoluminescence quenching, and Feng Xue for assistance with atomic layer deposition.

\section{Author contributions}

T.Z. and R.J.H. conceived and initiated the study. T.Z. carried out the experiments and data analysis. D.B.D. synthesized the CdSe QDs and performed atomic layer deposition of $\mathrm{HfO}_{2}$. T.Z. and R.J.H. prepared the manuscript. T.Z., D.B.D., V.E.F. and R.J.H. contributed to the revision and evaluation of the manuscript.

\section{Additional information}

Supplementary Information accompanies this paper at https://doi.org/10.1038/s41467019-09062-8.

Competing interests: The authors declare no competing interests.

Reprints and permission information is available online at http://npg.nature.com/ reprintsandpermissions/

Journal peer review information: Nature Communications thanks the anonymous reviewers for their contribution to the peer review of this work.

Publisher's note: Springer Nature remains neutral with regard to jurisdictional claims in published maps and institutional affiliations.

\begin{abstract}
(c) (i) Open Access This article is licensed under a Creative Commons Attribution 4.0 International License, which permits use, sharing, adaptation, distribution and reproduction in any medium or format, as long as you give appropriate credit to the original author(s) and the source, provide a link to the Creative Commons license, and indicate if changes were made. The images or other third party material in this article are included in the article's Creative Commons license, unless indicated otherwise in a credit line to the material. If material is not included in the article's Creative Commons license and your intended use is not permitted by statutory regulation or exceeds the permitted use, you will need to obtain permission directly from the copyright holder. To view a copy of this license, visit http://creativecommons.org/ licenses/by/4.0/.
\end{abstract}

(C) The Author(s) 2019 